\title{
Fueling the central engine of radio galaxies
}

\section{The molecular/dusty disk of $4 \mathrm{C} 31.04^{\star}$}

\author{
S. García-Burillo ${ }^{1}$, F. Combes ${ }^{2}$, R. Neri ${ }^{3}$, A. Fuente ${ }^{1}$, A. Usero ${ }^{1,4}$, S. Leon ${ }^{5}$, and J. Lim ${ }^{6}$
}

1 Observatorio Astronómico Nacional, Alfonso XII, 3, 28014 Madrid, Spain

e-mail: s.gburillo@oan.es, a.fuente@oan.es

2 Observatoire de Paris, LERMA, 61 Av. de l'Observatoire, 75014 Paris, France

e-mail: francoise.combes@obspm.fr

3 Institut de Radioastronomie Millimétrique, 300 rue de la Piscine, 38406 St. Martin d'Hères, France e-mail: neri@iram.fr

${ }^{4}$ Centre for Astrophysics Research, University of Hertfordshire, College Lane, AL10 9AB, UK

e-mail: a.usero@herts.ac.uk

5 Instituto de Astrofísica de Andalucía, C ${ }^{\circ}$ Bajo de Huétor, 50, 18008 Granada, Spain e-mail: stephane@iaa.es

${ }^{6}$ Inst. of Astron. and Astrophysics, Academia Sinica, PO Box 23-141, Taipei 106, Taiwan

e-mail: jlim@asiaa.sinica.edu.tw

Received 3 April 2007 / Accepted 25 April 2007

\section{ABSTRACT}

\begin{abstract}
We report the detection of a massive $\left(M_{\mathrm{gas}}>5 \times 10^{9} M_{\odot}\right)$ molecular/dusty disk of $1.4 \mathrm{kpc}$-size fueling the central engine of the compact symmetric object (CSO) 4C 31.04, based on high-resolution $\left(0.5^{\prime \prime}-1.2^{\prime \prime}\right)$ observations done with the IRAM Plateau de Bure interferometer (PdBI). These observations allow us for the first time to detect and map the continuum emission from dust at $218 \mathrm{GHz}$ in the disk of a CSO. The case for a massive disk is confirmed by detection of strong $\mathrm{HCO}^{+}(1-0)$ line emission and absorption. The molecular gas mass of $4 \mathrm{C} 31.04$ is in the range $0.5 \times 10^{10}-5 \times 10^{10} M_{\odot}$. While the distribution and kinematics of the gas roughly correspond to those of a rotating disk, we find evidence of distortions and non-circular motions that suggest the disk is not in a dynamically relaxed state. We discuss the implications of these results for the understanding of the evolution of radio galaxies.
\end{abstract}

Key words. galaxies: individual: 4C 31.04 - galaxies: ISM - galaxies: kinematics and dynamics - galaxies: active - galaxies: nuclei

\section{Introduction}

In constrast to the well-developed radio jets of classical radio galaxies (on scales of $\sim$ a few $10-100 \mathrm{kpc}$ ), the distinguishing property of compact symmetric objects (CSOs) is the small separation between their radio lobes ( a few $100 \mathrm{pc}-1 \mathrm{kpc})$. Two scenarios have been proposed to account for the radio structure of CSOs: the youth scenario, which contemplates CSOs as young radio galaxies with jets in the making (e.g. Fanti et al. 1995; Owsianik \& Conway 1998), and the frustration scenario, which interprets the absence of large jets as a consequence of the confinement by a highly dense, massive cocoon surrounding the central engine (e.g. O' Dea et al. 1991; Bicknell et al. 1997). Of particular note is the mounting evidence that interaction between the radio plasma and the ISM present in radio-loud galaxies is also at work in CSOs (Morganti et al. 2004) .

A study of the content, distribution, and kinematics of cold gas via molecular and atomic lines is the key to elucidating the nature of CSOs and their evolutionary link to other radio-loud galaxies. 4C 31.04 is a nearby CSO at $z \sim 0.06$, located at the

^ Based on observations carried out with the IRAM Plateau de Bure Interferometer. IRAM is supported by INSU/CNRS (France), MPG (Germany) and IGN (Spain). nucleus of the giant elliptical MCG 5-8-18. HI absorption was detected by Van Gorkom et al. (1989) and Mirabel (1990), and then mapped at high resolution by Conway (1996). The HST color maps obtained by Perlman et al. (2001)(Pe01) reveal that the optical image of 4C 31.04 is permeated with obscuration features. These consist of an elongated $\sim 1^{\prime \prime}$-central disk oriented along PA $\sim 5^{\circ}-10^{\circ}$ and surrounded by a spiral-like structure that stretches from the disk over the central $\sim 4^{\prime \prime}$ of the image. The central disk is roughly perpendicular to the axis of the double-lobe radio source shown in the $5 \mathrm{GHz}$ VLBA image of Giovannini et al. (2001) (Gi01). This radio source consists of two lobes that are separated by $\sim 0.07 "$ and extend close to the plane of the sky.

We have probed the gas and dust content of 4C 31.04 by observing the $\mathrm{HCO}^{+}(1-0)$ and ${ }^{12} \mathrm{CO}(2-1)$ molecular lines and their underlying continuum emission with unprecedented resolution $\left(\leq 1^{\prime \prime}\right)$ using the IRAM Plateau de Bure Interferometer (PdBI). In this Letter we discuss the results from analyzing the PdBI maps of continuum emission detected at $1 \mathrm{~mm}$ and $3 \mathrm{~mm}$. We also analyzed the $\mathrm{HCO}^{+}(1-0)$ map of the galaxy showing the emission and absorption of this line. Due to insufficient sensitivity, the $\mathrm{CO}(2-1)$ molecular line has only been detected towards the central offset of the galaxy. 


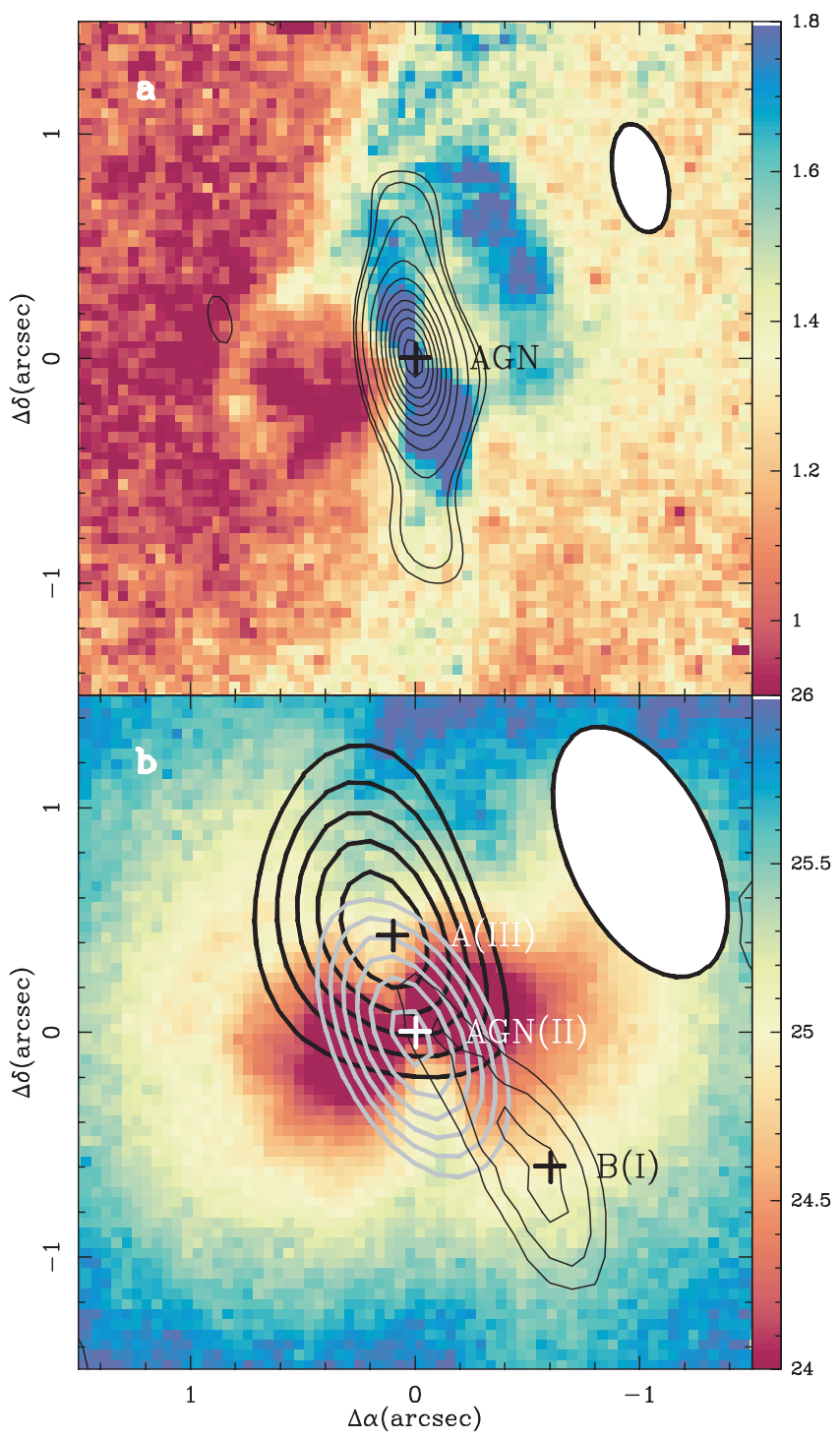

Fig. 1. a) The $1 \mathrm{~mm}$-continuum PdBI map (at $218 \mathrm{GHz}$ ) of $4 \mathrm{C} 31.04$ (contours: $3 \sigma, 4 \sigma, 7 \sigma$ to $55 \sigma$ in steps of $6 \sigma ; 1 \sigma=0.8 \mathrm{mJy}^{\text {beam }}{ }^{-1}$ ) is overlaid on the $R-H$ color image of Pe01 from HST (color scale). $(\Delta \alpha, \Delta \delta)$-offsets in arcsec are relative to the location of the AGN. b) The $\mathrm{HCO}^{+}(1-0)$ line maps of 4C 31.04 obtained after subtraction of the continuum source for the three velocity channels defined in Fig. 3: $\mathrm{I}(\mathrm{emission})=[-300,25] \mathrm{km} \mathrm{s}^{-1}$ (black thin contours; with maximum at B), II(absorption) $=[25,275] \mathrm{km} \mathrm{s}^{-1}$ (grey contours; with maximum at the AGN), III(emission) $=[275,900] \mathrm{km} \mathrm{s}^{-1}$ (black thick contours; with maximum at A). Levels are $3.5 \sigma, 4 \sigma$, and $4.5 \sigma$ for channel I $\left(1 \sigma(\mathrm{I})=0.06 \mathrm{Jy} \mathrm{km} \mathrm{s} \mathrm{km}^{-1}\right),-40 \sigma$, to $-100 \sigma$ in steps of $-10 \sigma$ for channel II $\left(1 \sigma(\mathrm{II})=0.05 \mathrm{Jy} \mathrm{km} \mathrm{s} \mathrm{s}^{-1}\right)$ and $5 \sigma$ to $13 \sigma$ in steps of $2 \sigma$ for channel III $\left(1 \sigma(\mathrm{III})=0.08 \mathrm{Jy} \mathrm{km} \mathrm{s}^{-1}\right)$. $\mathrm{HCO}^{+}$contours are overlaid on the F702W HST image of $\mathrm{Pe} 01$ (color scale in mag pixel ${ }^{-1}$ ). Ellipses represent the beams at $218 \mathrm{GHz}$ (a)) and $84 \mathrm{GHz}$ (b)). See Sect. 3 for details.

\section{Observations}

We observed 4C 31.04 simultaneously in the $\mathrm{HCO}^{+}(1-0)$ and ${ }^{12} \mathrm{CO}(2-1)$ transitions with the $\mathrm{PdBI}$ in the $\mathrm{A}+$ configuration on February 2006. We used one receiver setting centered on the $\mathrm{HCO}^{+}$transition (redshifted to $84.204 \mathrm{GHz}$ ) and two overlapping settings (centered each at $217.433 \mathrm{GHz}$ and $217.873 \mathrm{GHz}$ ) to sample the ${ }^{12} \mathrm{CO}(2-1)$ line (redshifted to $217.653 \mathrm{GHz}$ ). These receiver settings translate into a velocity coverage of $\sim 2000 \mathrm{~km} \mathrm{~s}^{-1}$ at $84 \mathrm{GHz}$ and $\sim 1400 \mathrm{~km} \mathrm{~s}^{-1}$ at $218 \mathrm{GHz}$. The phase-tracking center was set at $\left(\alpha_{2000}, \delta_{2000}\right)=\left(01^{\mathrm{h}} 19^{\mathrm{m}} 35.001^{\mathrm{s}}\right.$, $\left.32^{\circ} 10^{\prime} 50.06^{\prime \prime}\right)$, coincident with the position of the radiocontinuum source core (Gi01). The line visibilities at $84 \mathrm{GHz}$ were self-calibrated on the point-like radio-continuum source in the $84.402-84.475 \mathrm{GHz}$ band. The $1 \sigma$-noise for an on-source integration time of $9 \mathrm{~h}$ is $0.6 \mathrm{mJy}^{-1}$ beam $^{-1}$ in $7.5 \mathrm{MHz}$-channels. At $218 \mathrm{GHz}$ the visibilities were calibrated using the antennabased scheme. The point-source sensitivity at $218 \mathrm{GHz}$ is $1.9 \mathrm{mJy} \mathrm{beam}^{-1}$ in $15 \mathrm{MHz}$-channels. Maps were obtained with uniform weighting and yielded synthesized beams of $0.50^{\prime \prime} \times$ $0.24^{\prime \prime} @ \mathrm{PA}=12^{\circ}$ at $218 \mathrm{GHz}$ and 1.20" $\times 0.64^{\prime \prime} @ \mathrm{PA}=26^{\circ}$ at $84 \mathrm{GHz}$. We obtained a map of the continuum emission at $218 \mathrm{GHz}$ in $4 \mathrm{C} 31.04$ using the $1 \mathrm{GHz}$-wide image side band of the $1 \mathrm{~mm}$ receivers. Inside this window, there is no contribution from line emission. Similarly, a $3 \mathrm{~mm}$ continuum map (at $84 \mathrm{GHz}$ ) was built using velocity channels free of line emission in the signal side band of the PdBI receivers. The corresponding $1-\sigma$ sensitivities are $\sim 0.8 \mathrm{mJy}^{-1}$ beam $^{-1}$ at $218 \mathrm{GHz}$ and $\sim 0.5 \mathrm{mJy}_{\text {beam }}{ }^{-1}$ at $84 \mathrm{GHz}$. Although re-determined in this work, the velocity scale initially refers to the redshift $z=0.0592$ derived by Sargent (1973). Luminosity and angular distances are $D_{\mathrm{L}}=260 \mathrm{Mpc}$ and $D_{\mathrm{A}}=230 \mathrm{Mpc}$; the latter gives $1^{\prime \prime}=1.1 \mathrm{kpc}$.

\section{Detection of a molecular/dusty disk}

The $1 \mathrm{~mm}$ map shown in Fig. 1a reveals a strong emission source in the nucleus of 4C 31.04. The source can be decomposed into an unresolved central component and a disk. The disk extends over $\sim 1.6^{\prime \prime}(\sim 1.8 \mathrm{kpc})$ along PA $\sim 5^{\circ}-10^{\circ}$. The measured $1 \mathrm{~mm}-$ continuum visibilities can be fitted by a $\sim 40 \mathrm{mJy}$ point source centered on the AGN and an elliptical Gaussian source, which has a deconvolved $F W H M$-size of $1^{\prime \prime} \times 0.1^{\prime \prime}(1.1 \mathrm{kpc} \times 100 \mathrm{pc})$ and an integrated flux of $\sim 20 \mathrm{mJy}$. The continuum emission at $3 \mathrm{~mm}$ consists of a point source of $\sim 160 \mathrm{mJy}$ with no extended disk detected to the limits of our spatial resolution and sensitivity. The point source detected at $1 \mathrm{~mm}$ and $3 \mathrm{~mm}$ is the higher-frequency counterpart of the non-thermal double-lobe radio source mapped at cm-wavelengths in this CSO (e.g., Gi01). The spectral index $(\alpha)$ of the total continuum emission between $84 \mathrm{GHz}$ and $218 \mathrm{GHz}$ (with $S_{v} \sim v^{-\alpha}$ ) confirms the dominance of the non-thermal central component: $\alpha \geq 1$.

The detected $1 \mathrm{~mm}$ disk is tightly linked to the dusty disk identified in the $R-H$ color image of Pe01 (see Fig. 1a). The dusty disk seems to be mostly edge-on and oriented perpendicular to the axis of the double-lobe radio source. However, thermal free-free emission from an embedded star-forming episode could also partly contribute to the disk emission at $1 \mathrm{~mm}$. While there is no evidence of such a disk at $\mathrm{cm}$ wavelengths, it should be noted that the published VLBA/VLBI maps of 4C 31.04 would not be able to recover any emission on the typical scales of the disk. However the non-detection of an extended disk at $3 \mathrm{~mm}$ argues against a significant free-free emission component at $1 \mathrm{~mm}$. Furthermore, there is no detection of a thermal outflowlike component either at $1 \mathrm{~mm}$ or at $3 \mathrm{~mm}$.

If the $1 \mathrm{~mm}$ flux of the disk can be attributed to dust emission, a mass budget $\left(M_{\text {dust }}\right)$ can be derived provided that values for the temperature $\left(T_{\text {dust }}\right)$ and for the emissivity of dust $(\kappa)$ are assumed. The IRAS observations reveal significant amounts of warm dust in $4 \mathrm{C} 31.04$. From the flux ratio measured by IRAS $S_{v}(100 \mu) / S_{v}(60 \mu) \sim 3.5$, we derive a range of $T_{\text {dust }}=25-$ $30 \mathrm{~K}$ for a corresponding range of $\beta=2-1$ in the wavelength dependency of $\kappa \sim \lambda^{-\beta}$. Taking $\kappa(850 \mu)=0.9 \mathrm{~cm}^{2} \mathrm{gr}^{-1}$ from 


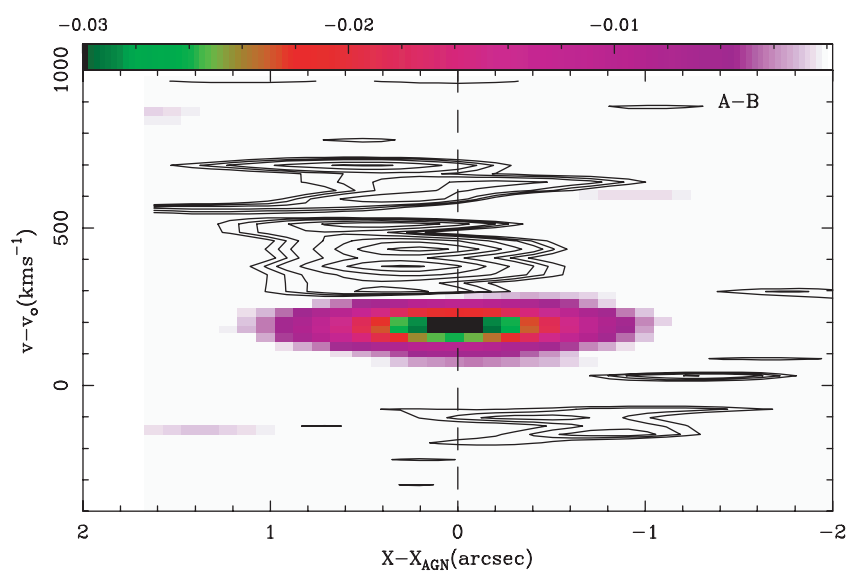

Fig. 2. The kinematics of molecular gas along the strip connecting points $\mathrm{A}$ and $\mathrm{B}$ (emission peaks for $\mathrm{HCO}^{+}(1-0)$ ) are illustrated by a position-velocity diagram. Contour levels represent emission of $\mathrm{HCO}^{+}(1-0): 2 \sigma, 2.5 \sigma, 3 \sigma$ to $7 \sigma$ in steps of $1 \sigma ; 1 \sigma=0.6 \mathrm{mJy}^{-1}$ beam $^{-1}$. Color scale $\left(\mathrm{mJy} \mathrm{beam}^{-1}\right)$ represents absorption in $\mathrm{HCO}^{+}(1-0)$ near the AGN. X-offsets are in arcsec relative to the position closest to the AGN. The velocity scale in $Y$ axis is relative to $v_{0}(z=0.0592)$.

Klaas et al. (2001) and $T_{\text {dust }}=30 \mathrm{~K}$ (i.e., the solution for $\beta=1$ ), we derive $M_{\text {dust }} \sim 6 \times 10^{7} M_{\odot}$ for the warm dust. To better estimate the total dust mass we have fitted the fluxes measured by IRAS (at $100 \mu$ and $60 \mu$ ) and the PdBI (at $1.3 \mathrm{~mm}$ ) using a twocomponent model for the dust temperatures. Any plausible fit to the observations gives a value for $M_{\text {dust }}$ of (4-7) $\times 10^{8} M_{\odot}$. The higher (lower) value of $M_{\text {dust }}$ corresponds to the fit with $\beta=1.5$ $(\beta=1)$ and temperatures of $\sim 16 \mathrm{~K}(21 \mathrm{~K})$ and $\sim 32 \mathrm{~K}(39 \mathrm{~K})$ for the cold and warm dust components, respectively. The cold dust component seems to contain the bulk of the mass: $M_{\text {dust }}^{\text {cold }}>$ $0.95 \times M_{\text {dust }}^{\text {total }}$.

Taking a molecular gas-to-dust ratio $\sim 100$ (Seaquist et al. 2004), we foresee that the total molecular gas mass of the disk might be $M_{\text {gas }} \sim(4-7) \times 10^{10} M_{\odot}$. If we allow for a minor contribution of free-free emission to the $1 \mathrm{~mm}$ flux, this can be taken as an upper limit to $M_{\text {gas }}$. As argued in Sect. 3, the case for a massive gas reservoir in 4C 31.04 is supported by the detection of $\mathrm{HCO}^{+}(1-0)$ emission from a rotating molecular disk.

\subsection{Distribution and kinematics}

The channel maps obtained after subtraction of the $3 \mathrm{~mm}$ continuum source show that $\mathrm{HCO}^{+}(1-0)$ is detected both in emission and absorption in 4C 31.04. As shown in Fig. $1 \mathrm{~b}, \mathrm{HCO}^{+}(1-$ $0)$ is detected in emission in two velocity ranges: from -300 to $25 \mathrm{~km} \mathrm{~s}^{-1}$ and from 275 to $900 \mathrm{~km} \mathrm{~s}^{-1}$ (channels I and III, respectively; see Fig. 3). The strongest emission (III) peaks NE of the AGN (offset $A=\left[0.1^{\prime \prime}, 0.4^{\prime \prime}\right]$ in Fig. 1b). Compared to III, emission in $\mathrm{I}$ is $\geq 3$ times weaker, and it peaks SW of the AGN (offset $\mathrm{B}=\left[-0.6^{\prime \prime},-0.6^{\prime \prime}\right]$ in Fig. 1b). In contrast, the line is detected in absorption from 25 to $275 \mathrm{~km} \mathrm{~s}^{-1}$ (channel II; see Fig. 3). The $\mathrm{HCO}^{+}$absorption is detected as a point source centered on the AGN. These results indicate that the distribution of molecular gas traced by $\mathrm{HCO}^{+}$has been spatially resolved.

To the first order, the emission and absorption of $\mathrm{HCO}^{+}$seem to arise from a rotating disk of $\sim 1.4 \mathrm{kpc}$-size. This is illustrated in Fig. 2, which shows the position-velocity $(p-v)$ plot along the strip connecting $\mathrm{A}$ and $\mathrm{B}$, i.e., close to the apparent kinematic major axis of the disk. By assuming that the total width (FWZP)

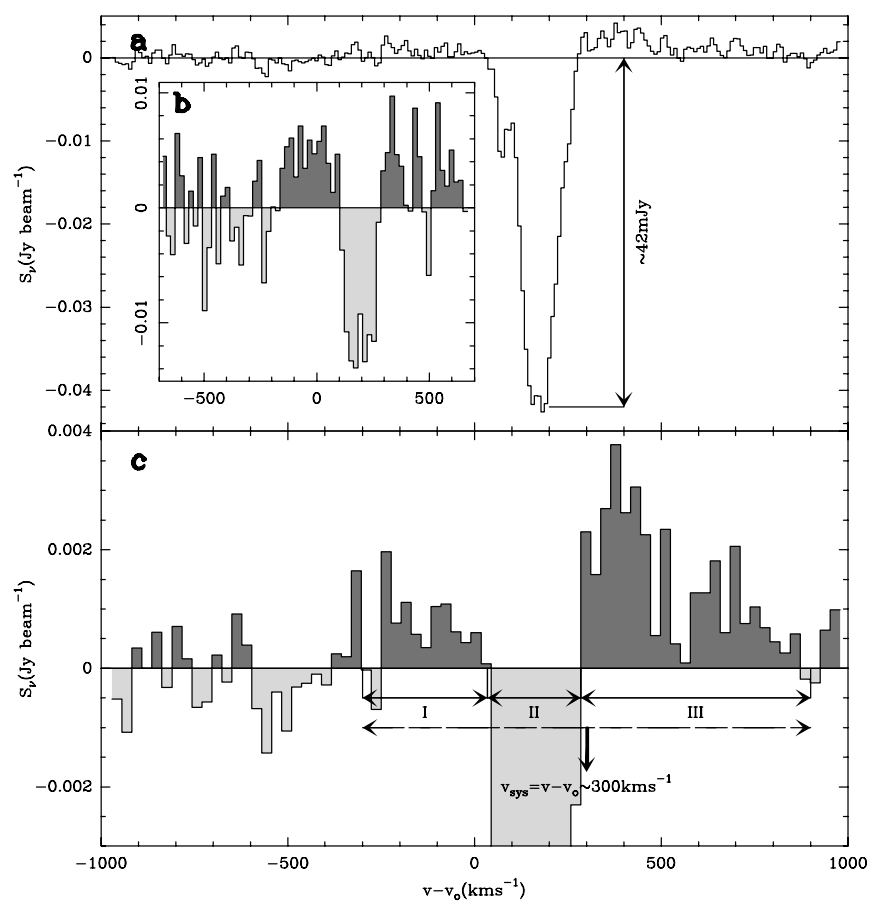

Fig. 3. a) Spectrum of the $\mathrm{HCO}^{+}(1-0)$ line emission and absorption at the central offset (AGN) of 4C 31.04. A strong absorption line is detected against the $3 \mathrm{~mm}$ continuum source. Velocity scale is relative to $v_{\mathrm{o}}$, which corresponds to $z=0.0592$. b) Same as a) but for the $\mathrm{CO}(2-$ 1) line. c) A zoomed view on the spectrum shown in a) helps to delimit intervals I-to-III and derive a new value for $v_{\text {sys }}$.

of the $\mathrm{HCO}^{+}$emission profile is twice the projected rotational velocity $\left(v_{\text {rot }}\right)$, we derive $v_{\text {rot }} \sim 500 \mathrm{~km} \mathrm{~s}^{-1}$ for an edge-on disk geometry and a value for the systemic velocity $\left(v_{\text {sys }}\right)$ that is redshifted by $+300 \mathrm{~km} \mathrm{~s}^{-1}$ with respect to the redshift initially assumed in this work $(z=0.0592)$. The $\mathrm{HCO}^{+}$-based redshift $\left(z\left(\mathrm{HCO}^{+}\right)=0.0602 \pm 0.0002\right)$ agrees with the values recently derived from the optical spectroscopy observations of 4C 31.04 $(z=0.0600 \pm 0.0001$; Marcha et al. 1996).

If circular motions completely ruled the gas kinematics, the absorption profile against the AGN (this being the dynamical center) should be centered at $v_{\text {sys }}$. This is, however, at odds with observations: as shown in Fig. 3, the gas seen in absorption in channel II is blue-shifted $\sim 150 \mathrm{~km} \mathrm{~s}^{-1}$ on average with respect to $z=0.0602$ (similar to the HI absorption of Conway 1996). This result can only be reconciled if the gas causing the absorption is outflowing from the nucleus or subject to strong noncircular motions, thus partly invalidating the initial premises. An alternative solution adopting the center of the absorption profile as $v_{\text {sys }}$ would only enhance the asymmetry in the $\mathrm{HCO}^{+}$ emission profile. Furthermore, there is evidence of distortions in the molecular disk from the observed distribution. The SW side of the molecular disk is offset from the dust disk: the $\mathrm{HCO}^{+}$ disk is tilted towards a larger PA (Fig. 1b). A conspiracy of absorption and emission may explain the observed anticorrelation. However, independent evidence is also found of distortions on the opposite side of the disk: whereas the bulk of the $\mathrm{HCO}^{+}$emission NE follows the dust disk along $\mathrm{PA}=5^{\circ}-10^{\circ}$, the molecular disk thickens along its minor axis in this region (Fig. 1b).

While the reasons explaining the observed distortions in the distribution and kinematics of the gas cannot be elucidated with 
these observations, these results further illustrate that the rotating molecular disk of 4C 31.04 is not dynamically relaxed.

\subsection{Masses}

The $\mathrm{HCO}^{+}$line in emission probes the dense phase $\left(n\left(\mathrm{H}_{2}\right)>\right.$ $10^{4} \mathrm{~cm}^{-3}$ ) of the molecular disk of 4C 31.04. Without further constraints at hand, an estimate of the mass of dense molecular gas $\left(M_{\text {dense }}\right)$ can be derived from the luminosity of the line $\left(L\left[\mathrm{HCO}^{+}\right]=4.2 \times 10^{8} \mathrm{~K} \mathrm{~km} \mathrm{~s}^{-1} \mathrm{pc}^{2}\right)$ if we assume low opacities, LTE conditions, an abundance for $\mathrm{HCO}^{+}\left(X\left[\mathrm{HCO}^{+}\right]\right)$, and an excitation temperature $\left(T_{\mathrm{ex}}\right)$ for the line. For $T_{\mathrm{ex}}=10 \mathrm{~K}$ and $X\left[\mathrm{HCO}^{+}\right]=10^{-8}$, we derive $M_{\text {dense }} \sim 5 \times 10^{8} M_{\odot}$. This is probably a lower limit, because the $\mathrm{HCO}^{+}$line is probably optically thick (see Fig. 3). Alternatively, we can derive $M_{\text {dense }}$ via an $L\left(\mathrm{HCO}^{+}\right)$-to- $M_{\text {dense }}$ conversion factor, if we assume that the emission comes from a clumpy medium of optically-thick virialized clouds. The value of the conversion factor would depend on the physical parameters of the clouds through the ratio $\sim n^{1 / 2}\left(\mathrm{H}_{2}\right) / T_{\mathrm{b}}\left(\mathrm{HCO}^{+}\right)$, where $n\left(\mathrm{H}_{2}\right)$ is the molecular gas density and $T_{\mathrm{b}}\left(\mathrm{HCO}^{+}\right)$the emergent linebrightness temperature of the clouds. Through LVG simulations, assuming a range for $T_{K}=20$-to- $60 \mathrm{~K}$ and $n\left(\mathrm{H}_{2}\right)=$ $10^{4}$-to- $10^{5} \mathrm{~cm}^{-3}$, we derive an average conversion factor of $M_{\text {dense }} / L\left(\mathrm{HCO}^{+}\right) \sim 10 M_{\odot}\left(\mathrm{K} \mathrm{km} \mathrm{s}^{-1} \mathrm{pc}^{2}\right)^{-1}$. This would imply that $M_{\text {dense }} \sim 4 \times 10^{9} M_{\odot}$. Taking $\mathrm{HCO}^{+}(1-0) / \mathrm{CO}(1-0)=0.05$ (Graciá-Carpio et al. 2006), we derive $M_{\text {gas }} / M_{\text {dense }} \sim 10$ and thus estimate $M_{\text {gas }}$ to range between $\sim 5 \times 10^{9} M_{\odot}$ and $\sim 4 \times 10^{10} M_{\odot}$.

The sensitivity reached in $\mathrm{CO}(2-1)$ was insufficient for mapping the emission of this line in $4 \mathrm{C} 31.04$, therefore $M_{\text {gas }}$ cannot be derived from these data. The line is detected in absorption and emission only towards the AGN (Fig. 3). However, Ocaña-Flaquer et al. (in prep.) have recently detected the emission and absorption of $\mathrm{CO}(1-0)$ and $\mathrm{CO}(2-1)$ in $4 \mathrm{C} 31.04$ using the IRAM $30 \mathrm{~m}$ telescope, improving on previous searches for $\mathrm{CO}$ emission (O'Dea et al. 2005; Evans et al. 2005). With COto- $\mathrm{H}_{2}$ conversion factor $N\left(\mathrm{H}_{2}\right) / I_{\mathrm{CO}}=2.2 \times 10^{20} \mathrm{~cm}^{-2} \mathrm{~K}^{-1} \mathrm{~km}^{-1} \mathrm{~s}$ (Solomon \& Barrett 1991), Ocaña-Flaquer et al. estimate $M_{\text {gas }} \sim$ $5 \times 10^{9} M_{\odot}$, a value within the range derived from $\mathrm{HCO}^{+}$. Note that, due to the superposition of absorption and emission in the profiles, the values derived from molecular line emission underestimate $M_{\text {gas }}$. Taken the estimates from dust continuum and line emission together, we conclude that $M_{\text {gas }}$ lies in the range $(0.5-5) \times 10^{10} M_{\odot}$. This is much higher than the mass of HI derived by Conway $(1996)\left(\sim 10^{8} M_{\odot}\right)$.

\subsection{Line absorption}

Assuming LTE conditions, we can derive the column densities of $\mathrm{HCO}^{+}\left(N_{\mathrm{HCO}^{+}}\right)$and $\mathrm{CO}\left(N_{\mathrm{CO}}\right)$ from the the $J \rightarrow J+1$ absorption lines detected towards the center of 4C 31.04 according to:

$N_{\text {mol }}=\frac{8 \pi v^{3}}{c^{3} A_{\mathrm{ul}} g_{\mathrm{u}}} \frac{Q\left(T_{\mathrm{ex}}\right) \exp \left(E_{J} / k T_{\mathrm{ex}}\right)}{\left(1-\exp \left(-h v / k T_{\mathrm{ex}}\right)\right)} \int \tau \mathrm{d} V$

where $E_{J}$ is the energy of the lower level of the transition, $T_{\text {ex }}$ the excitation temperature, $Q\left(T_{\mathrm{ex}}\right)=\sum_{J i}(2 J i+1) \exp \left(-E_{J i} / k T_{\mathrm{ex}}\right)$ the partition function, and $A_{\mathrm{ul}}$ the Einstein coefficient of the line. A lower limit to the velocity-integrated optical depth of the line $\int \tau \mathrm{d} V$, and thus to $N_{\text {mol }}$, can be derived by assuming that the covering factor $f$ of the absorbing molecular gas with respect to the extent of the radio source in 4C 31.04 is unity. In this case we replace $\int \tau \mathrm{d} V$ by $\tau_{\text {obs }} \Delta v$, where $\tau_{\mathrm{obs}}=\ln \left(T_{\mathrm{mb}}^{\mathrm{C}} / T_{\mathrm{mb}}^{\mathrm{L}}\right)$ with
$T_{\mathrm{mb}}^{\mathrm{C}}$ and $T_{\mathrm{mb}}^{\mathrm{L}}$ are the main brightness temperature of the continuum and the line, respectively, and $\Delta v$ the Gaussian width of the line. From $\tau_{\text {obs }}$ we estimate a lower limit to $f$ that is similar for the two lines $\left(f>1-\mathrm{e}^{-\tau_{\text {obs }}} \sim 0.27\right)$, suggestive of a common value for $f$. Assuming $f=1$ and $T_{\mathrm{ex}}=10 \mathrm{~K}$ for the two lines, we derive $N\left(\mathrm{HCO}^{+}\right) / N(\mathrm{CO})=1.6 \times 10^{-3}$. While this value is surprisingly high compared to galactic standards (see Table 3 of Wiklind \& Combes 1995, Wi95), it probably overestimates the true $\mathrm{HCO}^{+} / \mathrm{CO}$ abundance ratio for two reasons. First, $T_{\mathrm{ex}}$ is probably lower for the $\mathrm{HCO}^{+}$line than for the $\mathrm{CO}$ line. Second, the chances of the $\mathrm{CO}$ absorption being underestimated due to corruption by emission within the beam are much higher than for the $\mathrm{HCO}^{+}$absorption (see discussion in Wi95).

\section{Conclusions}

The high-resolution and high-sensitivity capabilities of the PdBI have allowed us to detect and map a massive molecular/dusty disk fueling the central engine of a CSO like 4C 31.04 for the first time. Based on the estimates derived from the continuum and line emissions, the molecular disk mass $M_{\text {gas }}$ lies in the range $(0.5-5) \times 10^{10} M_{\odot}$. Even if we adopt the lower bound to $M_{\text {gas }}$, these observations reveal the existence of significant amounts of molecular gas in the central $1.4 \mathrm{kpc}$ disk of 4C 31.04 , comparable to those identified in ULIRGs $\left(\sim 10^{9}-5 \times 10^{10} M_{\odot}\right.$; Sanders et al. 1991), and much larger than the amounts of cold gas detected in normal elliptical galaxies $\left(\sim 10^{7}-10^{8} M_{\odot}\right.$; Lees et al. 1991). Of particular note is that the high value of $M_{\text {gas }}$ derived for 4C 31.04 would be roughly consistent with the scenario of confinement in this radio source. Using simple analytical models, Carvalho (1998) predicts that a clumpy medium with $M_{\text {gas }} \sim 10^{9}-10^{10} M_{\odot}$ can confine a radio source on the scale of $0.5-1 \mathrm{kpc}$. In 4C 31.04 , the bulk of $M_{\text {gas }}$ is seen to be in a disk roughly perpendicular to the radio source's axis. This particular geometry would decrease the efficiency of frustration, however.

Within the frustration scheme, it is foreseen that a disturbed ISM around the radio AGN would reflect the interaction between the radio plasma and the dense confining cocoon. In the case of $4 \mathrm{C} 31.04$, there is evidence that a radio lobe-ISM interaction may be at work. The HST image of Pe01 shows cone-like features aligned with the radio axis of the source (see Fig. 1b), the likely signature of gas shocked by the jet (e.g., Labiano et al. 2003). Moreover, the distribution and kinematics of molecular gas probed by the $\mathrm{HCO}^{+}$observations presented in this work illustrate that the detected rotating disk is not in a fully relaxed state. The revealed distortions may reflect that the disk is still settling after a merger or an event of gas accretion. Alternatively, the jet and the cone-like features may be interacting with the disk, thus producing the reported distortions.

Obtaining a better constraint for $M_{\text {gas }}$ in 4C 31.04, and in CSOs in general, is the key to exploring the nature of these sources. As illustrated in this work, the central gas disks of radio galaxies are very likely dominated by the molecular phase. In order to take full advantage of molecular line data, high spatial resolution and high-sensitivity observations are needed to at the least reduce the bias due to the confusion of absorption and emission in the beam.

Acknowledgements. We acknowledge economic support from the Spanish MEC and Feder funds under grant ESP2003-04957 and from SEPCT/MEC under grant AYA2003-07584. We thank E. Perlman for providing the HST data for 4C 31-04. 


\section{References}

Bicknell, G. V., Dopita, M. A., \& O’Dea, C. P. O. 1997, ApJ, 485, 112 Carvalho, J. C. 1998, A\&A, 329, 845

Conway, J. E. 1996, Extragalactic Radio Sources, IAU Symp., 175, 92 Evans, A. S., Mazzarella, J. M., Surace, J. A., et al. 2005, ApJS, 159, 197 Fanti, C., Fanti, R., Dallacasa, D., et al. 1995, A\&A, 302, 317

Giovannini, G., Cotton, W. D., Feretti, L., Lara, L., \& Venturi, T. 2001, ApJ, 552, 508

Graciá-Carpio, J., García-Burillo, S., Planesas, P., \& Colina, L. 2006, ApJ, 640, L135

Klaas, U., Haas, M., Müller, S. A., et al. 2001, A\&A, 379, 823

Labiano, A., O’Dea, C. P., Gelderman, R., et al. 2003, PASA, 20, 28

Lees, J. F., Knapp, G. R., Rupen, M. P., \& Phillips, T. G. 1991, ApJ, 379, 177

Marcha, M. J. M., Browne, I. W. A., Impey, C. D., \& Smith, P. S. 1996, MNRAS, 281,425
Mirabel, I. F. 1990, ApJ, 352, L37

Morganti, R., Oosterloo, T. A., Tadhunter, C. N., et al. 2004, A\&A, 424, 119

O'Dea, C. P., Baum, S. A., \& Stanghellini, C. 1991, ApJ, 380, 66

O'Dea, C. P., Gallimore, J., Stanghellini, C., Baum, S. A., \& Jackson, J. M. 2005, AJ, 129, 610

Owsianik, I., \& Conway, J. E. 1998, A\&A, 337, 69

Perlman, E. S., Stocke, J. T., Conway, J., \& Reynolds, C. 2001, AJ, 122, 536

Sanders, D. B., Scoville, N. Z., \& Soifer, B. T. 1991, ApJ, 370, 158

Sargent, W. L. W. 1973, ApJ, 182, L13

Seaquist, E., Yao, L., Dunne, L., \& Cameron, H. 2004, MNRAS, 349, 1428

Solomon, P. M., \& Barrett, J. W. 1991, Dynamics of Galaxies and Their Molecular Cloud Distributions, IAU Symp., 146, 235

van Gorkom, J. H., Knapp, G. R., Ekers, R. D., et al. 1989, AJ, 97, 708

Wiklind, T., \& Combes, F. 1995, A\&A, 299, 382 